\title{
modélisation du comportement thermomécanique d'un massif granitique - application à la simulation de l'expérience THM de Fanay-Augères
}

\author{
numerical modelling of the thermomechanical behaviour \\ of a granitic mass - application to the simulation of Fanay-Augères \\ THM experiment
}

\author{
A. REJEB, G. VOUILLE \\ Ecole Nationale Supérieure des Mines de Paris* \\ S. DERLICH \\ Commissariat à l'Energie Atomique **
}

Rev. Franç. Géotech. $n^{\circ} 53$, pp. 21-31 (octobre 1990)

\section{Résumé}

L'expérience Thermo-Hydro-Mécanique de Fanay-Augères a permis d'obtenir un ensemble de résultats précis et cohérents en ce qui concerne l'évolution de la température et de la déformation. Cette base de données constitue une référence précieuse pour juger de la validité des modélisations numériques du comportement thermomécanique d'un massif de granite fissuré,

Cet article fait le point de l'ensemble des travaux réalisés dans le cadre de cette expérience et présente les premiers résultats obtenus à l'aide de simulations numériques utilisant un modèle thermoélastique puis un modèle thermoplastique en milieu continu équivalent.

\section{Abstract}

The Fanay-Augères Thermo-Hydro-Mechanical in situ experiment has produced a whole set of accurate and consistent results regarding the evolution of temperature and deformation. This data base is a valuable reference to appraise the validity of the numerical modellings of the thermomechanical behaviour of a fractured granite mass.

This paper presents the work done within the scope of this experiment and gives the first results obtained by means of numerical simulations using at first a thermoelastic and then a thermoplastic model in an equivalent continuous medium. 


\section{INTRODUCTION}

L'enfouissement irréversible profond des déchets radioactifs de haute activité, émetteurs de chaleur, dans des formations géologiques stables et peu perméables aux eaux souterraines, est l'option considérée actuellement comme la plus réaliste en vue d'assurer leur isolation jusqu'à des niveaux d'activité inoffensifs visà-vis de la biosphère et de l'homme en particulier (CCE et OCDE-AEN, 1984).

Le concept d'évacuation est du type \& multi-barrières ", c'est-à-dire que les conteneurs, éventuellement enveloppés d'un suremballage métallique épais, sont déposés dans des dépôts minés profonds composés de galeries d'accès et de forages, reliés à la surface par des puits. L'ensemble est soigneusement remblayé de matériaux appropriés après mise en place de tous les conteneurs.

Les milieux-hôtes les plus fréquemment étudiés actuellement sont (a) les roches dures peu fracturées telles que le granite et le schiste, (b) les matériaux argileux pouvant montrer d'intéressantes propriétés de rétention vis-à-vis des radio-éléments, et (c) les formations salines, normalement exemptes de toute circulation de fluides. Le basalte étudié aux Etats-Unis s'apparente mécaniquement à la catégorie du granite (CÔME, 1988).

Pour quantifier expérimentalement les phénomènes thermo-hydro-mécaniques, la majorité des pays impliqués dans les projets d'évacuation de déchets radioactifs ont entrepris des programmes de recherche dans plusieurs "laboratoires souterrains". Les résultats de tels essais serviront aussi à caler les modèles numériques appropriés.

En ce qui concerne le stockage dans le granite, les plus importantes expérimentations ont été réalisées : - dans la mine de Stripa en Suède (OCDE-AEN, 1983) :

- sur les sites américains du Nevada (ZIMMERMAN, 1986) :

- dans le laboratoire souterrain du Grimsel en Suisse (CÔME et al., 1985) ;

- dans la mine de Fanay-Augères en Haute-Vienne en France (CEA/IPSN-SEPD, 1986).

La présente communication s'intéresse à l'expérience de Fanay-Augères; une modélisation du comportement thermomécanique du massif granitique considéré est proposée. Les nombreuses valeurs expérimentales obtenues in situ ont permis de tirer des conclusions sur la validité de cette modélisation.

\section{DESCRIPTION DE L'EXPÉRIENCE}

L'expérience Thermo-Hydro-Mécanique (THM) a été réalisée par l'Institut de Protection et de Sureté Nucléaire du Commissariat à l'Energie Atomique à la mine d'uranium de Fanay-Augères, exploitée par la
COGEMA dans le Limousin. Les dimensions et la durée de cette expérience à échelle réduite ont été déterminées par une similitude spatio-temporelle avec un stockage géologique réel à $1000 \mathrm{~m}$ de profondeur pendant un million d'années. Le laboratoire est situé à $100 \mathrm{~m}$ de profondeur; sous le radier, un volume instrumenté de dimensions $10 \times 10 \times 5 \mathrm{~m}$, est chauffé dans le but de caractériser les modifications du milieu sur les plans thermique, hydraulique et mécanique lors de ce chauffage et postérieurement. L'implantation des appareils de mesure a été effectuée selon un repère local $(\mathrm{X}, \mathrm{Y}, \mathrm{Z})$ qui délimite un maillage codé de la zone d'étude comme le montre la figure 1.

La source chauffante enfouie à $3 \mathrm{~m}$ sous la surface du radier est constituée de 5 radiateurs de $1,5 \mathrm{~m}$ de longueur et $0,15 \mathrm{~m}$ de diamètre. La puissance totale est de $1 \mathrm{~kW}$ soit 200 watts par élément chauffant. Les radiateurs ayant un entre-axe de $0,30 \mathrm{~m}$, sont disposés à l'intérieur de 5 forages parallèles et horizontaux de longueur $10 \mathrm{~m}$. On note que la puissance d'un élément chauffant de la source correspond à l'émission thermique d'un conteneur de déchets d'un volume de 1501 , d'un diamètre de $0,35 \mathrm{~m}$ et d'une hauteur de $1,50 \mathrm{~m}$ au bout d'une période de refroidissement de 30 ans (CCE, 1982).

Les mesures ont été poursuivies pendant environ 51 jours de chauffage, interrompus par quelques coupures de courant électrique, et 74 jours de refroidissement. Ces mesures, effectuées en particulier au niveau du radier ou dans des forages verticaux creusés à partir de celui-ci, avaient pour objectifs de contrôler :

- la répartition de la température à la surface du radier et dans le volume rocheux autour de la source de chaleur ;

- les déformations en surface et en profondeur en particulier au niveau des fractures naturelles;

- la variation de la conductivité hydraulique de quelques fractures initialement bien identifiées.

Toutes ces mesures ont été enregistrées et mémorisées par un dispositif d'acquisition automatique des données, piloté par micro-ordinateur. Les résultats obtenus nous ont été fournis par la société SIMECSOL qui a réalisé cette installation (GATHION et PINCENT, 1988).

\section{SYNTHÈSE DES RÉSULTATS DE MESURE}

Au niveau thermique, on a noté une bonne cohérence des mesures enregistrées par les capteurs de température. A la fin du chauffage, la source de chaleur a atteint une température maximale de $77^{\circ} \mathrm{C}$, alors que dans le massif rocheux, elle n'était que d'environ $41^{\circ} \mathrm{C}$. Les deux premières pannes de chauffage de durée 3 heures et 22 heures ont montré une influence significative sur les températures mesurées surtout par les capteurs les plus proches de la source. La courbe que présente la figure 2 est un exemple des résultats de mesures obtenus où appa- 


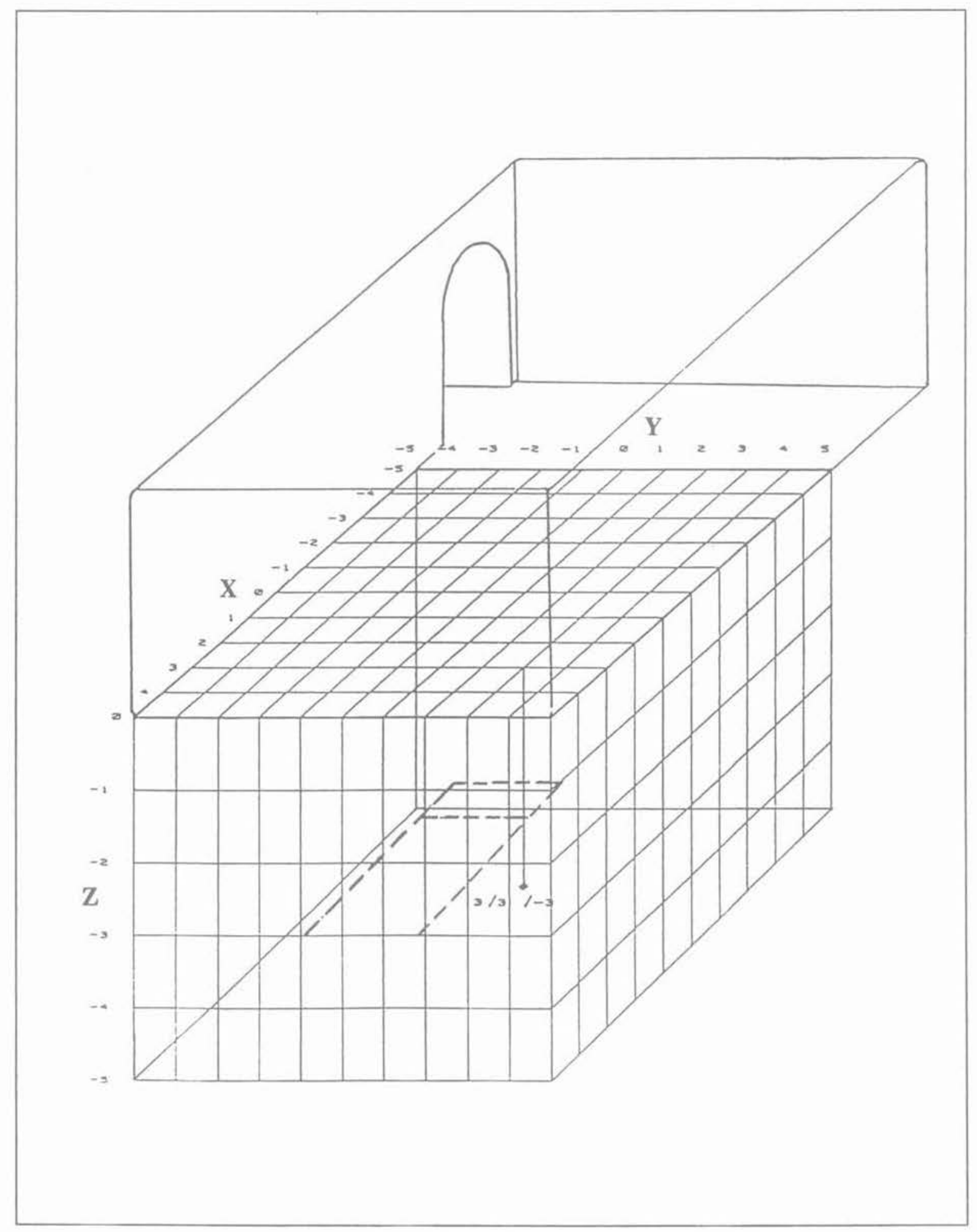

Fig. 1. - Schéma du laboratoire (d'après SIMECSOL).

Fig. 1. - Diagram of the laboratory (according to SIMECSOL).

raît clairement la présence de deux pics d'amplitude différente correspondant aux deux interruptions de chauffage. Les mesures des températures en surface ont montré que c'est uniquement le centre du radier qui s'est légèrement échauffé et que partout ailleurs les capteurs ont mesuré l'évolution de la température extérieure, décroissante de 13 à $10^{\circ} \mathrm{C}$.

Dans le but de dégager un éventuel effet de la fissuration et de l'hétérogénéité du milieu sur la réparti- 


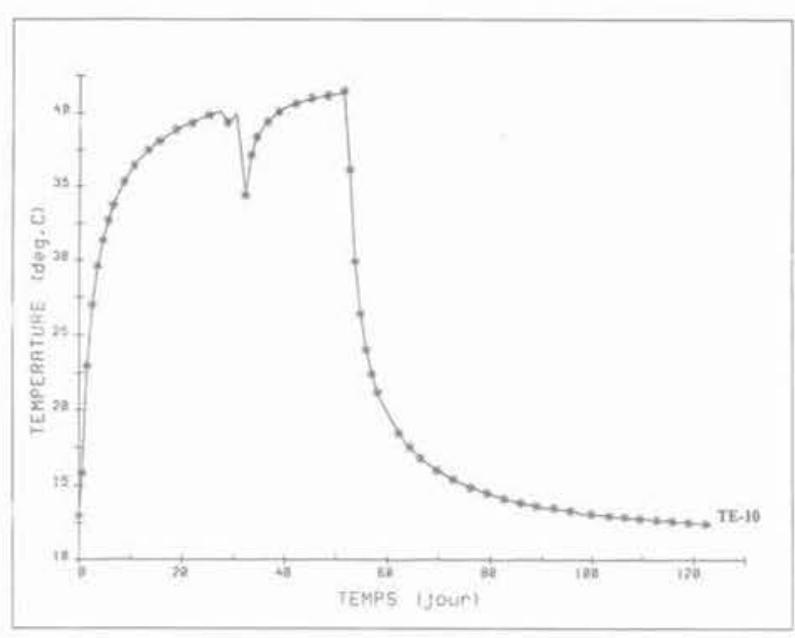

Fig. 2. - Résultats de mesures enregistrées par le capteur de température TE-10, de coordonnées locales $(0,-0,2,-2,5)$.

Fig. 2. - Results of the measurements recorded by the temperature transducer TE-70, with local co-ordinates $(0,-0.2,-2.5)$.

tion de la température dans le massif granitique, nous avons réalisé des isothermes dans différents plans horizontaux et dans les deux plans verticaux $X=0$ et $Y=0$ à diverses dates (REJEB et al., 1988). Le tracé de ces isothermes a été effectué en utilisant un programme de krigeage tridimensionnel développé au Centre de Géostatistique de l'ENSMP. La régularité des lignes isothermes obtenues permet d'écarter l'existence de singularité particulière des propriétés thermiques du massif rocheux. De plus, aux dates considérées, l'uniformité et la cohérence de la répartition du champ de température dans les deux plans $X=0$ et $Y=0$, ayant des traces de fissuration différentes, montrent que la présence des fissures n'a pas eu un effet important sur les mesures comme lillustre l'exemple de la figure 3 .

Les mesures de déformation en profondeur ont été effectuées à l'aide de 3 extensomètres de forage $\left(F_{1}\right.$. $F_{2}$, et $F_{3}$ ) comprenant chacun 4 capteurs de déplacement. Ces capteurs mesurent l'évolution des variations de distance entre le radier et leur point d'ancrage dans le forage au cours des périodes de chauffage et de refroidissement.

L'analyse des résultats de ces mesures a montré que le granite a répondu quasi-instantanément au chauffage puisqu'on n'a observé pratiquement aucun décalage dans le temps entre l'évolution des températures et celle des déplacements dans le massif. La figure 4 confirme la bonne cohérence de ces mesures avec celles des températures (fig, 2) et montre la haute sensibilité des instruments de mesure utilisés.

L'évolution de la déformation du radier selon les axes (X, Y,) a été suivie par les mesures des extensomètres électriques et manuels répartis de manière à couvrir presque la totalité de la surface du laboratoire.

Globalement, en fin de chauffage, la surface du radier s'est déformée tangentiellement de $0,2 \mathrm{~mm} / \mathrm{m}$ et radialement de $0,04 \mathrm{~mm} / \mathrm{m}$; les déformations tan-
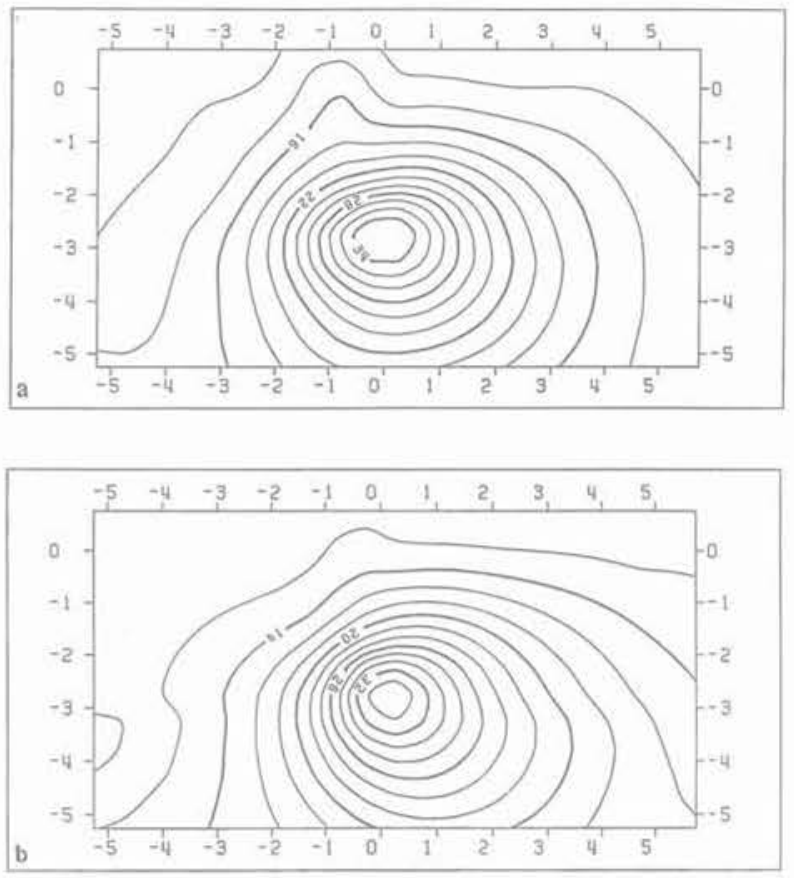

Fig. 3. - Isothermes expérimentales à la date 51 jours.

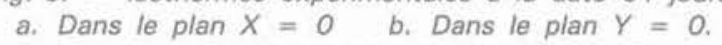

Fig. 3. - Experimental isothermals on day 57

a. In the plane $X=0 \quad$ b. In the plane $Y=0$.

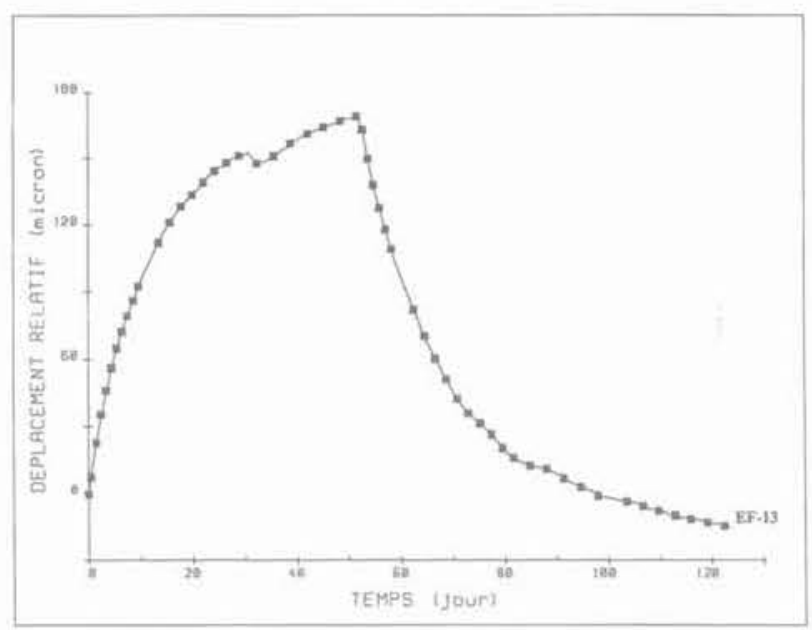

Fig. 4. - Resultats de mesures enregistrées par l'extensomètre de forage EF-13, de coordonnées locales $(-1,0,-4)$.

Fig. 4. - Results of the measurements recorded by the borehole extensometer EF-13, with local co-ordinates $(-1,0,-4)$.

gentielle et radiale sont définies par rapport à un repère polaire lié au centre du radier. Les mesures par un système de laser tournant ont mis en évidence un soulèvement du radier de quelques dixièmes de millimètre. La faible amplitude de ces déformations traduit le fait que l'échauffement du massif a été très limité, en particulier la température au voisinage de la source ne s'est élevée que d'environ $28^{\circ} \mathrm{C}$.

Les mesures extensométriques en surface, comme celles en profondeur, ont fait apparaître un comportement irréversible du granite vis-à-vis du chauffage. De 
plus, l'analyse des mesures de déformation du radier a permis d'identifier une influence significative de la fissuration ; en effet, des extensomètres placés symétriquement par rapport au centre du radier n'ont pas fourni les mêmes valeurs de déformation. Les valeurs obtenues ont été trouvées largement liées à la position des extensomètres par rapport aux fissures ainsi qu'à la densité de fracturation de la zone considérée (REJEB et al., 1989). En prenant l'exemple des extensomètres électriques ET-4, ET-8, EL-13 et EL-15 placés tangentiellement à $2 \mathrm{~m}$ du centre de radier (fig. 5), on observe qu'en passant de EL-15, se trouvant dans une zone relativement saine, à ET-8 placé à cheval sur la faille 8 (fig. 6), les déformations tangentielles se trouvent multipliées par un facteur de lordre de 6.

Par ailleurs, les deux fissures principales du radier ont été aussi instrumentées (fig. 7) afin de contrôler l'évolution de leur ouverture et de leur rejet. A titre d'exemple, la figure 8 donne les mesures enregistrées sur la fissure 2 par un extensomètre électrique horizontal et un extensomètre vertical. On note que la fissure 2 avait une ouverture initiale avant chauffage de $4 \mathrm{~mm}$ et que la base de mesure était de $50 \mathrm{~cm}$. On constate qu'à la fin de l'expérience, cette fissure s'est refermée seulement de $31 \%$ de son ouverture maximale.

\section{MODÈLES NUMÉRIQUES}

Les outils numériques utilisés pour les simulations sont les logiciels $\mathrm{CHEF}$ (CHaleur par Eléments Finis) et VIPLEF (VIsco-PLasticité par Eléments Finis), développés au Centre de Géotechnique et d'Exploitation du Sous-sol de l'ENSMP (TIJANI, 1985) ; ils permettent le couplage thermomécanique en utilisant le même maillage.

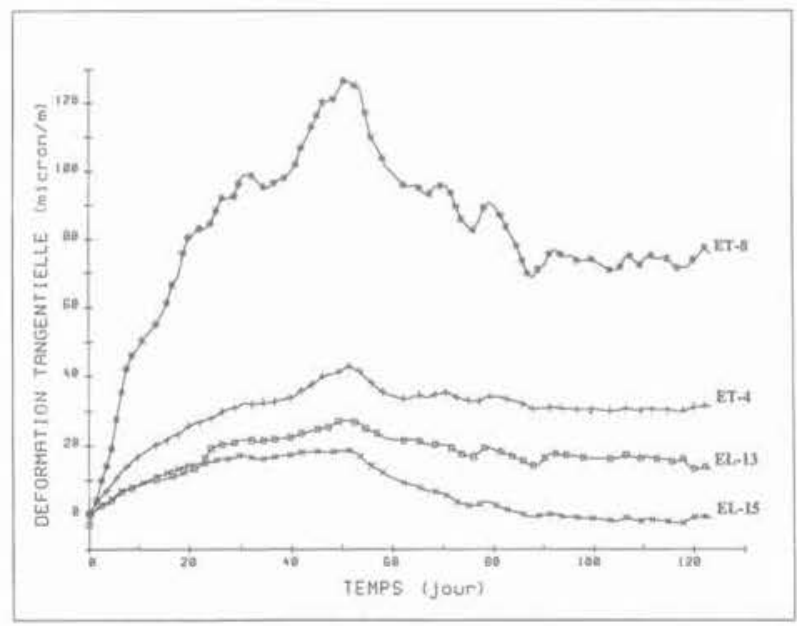

Fig. 5. - Résultats de mesures enregistrées par les extensomètres de surface ET-4, ET-8, EL-13 et EL-15. Fig. 5. - Results of the measurements recorded by the surface extensometers ET-4, ET-8, EL-13 and EL-15.

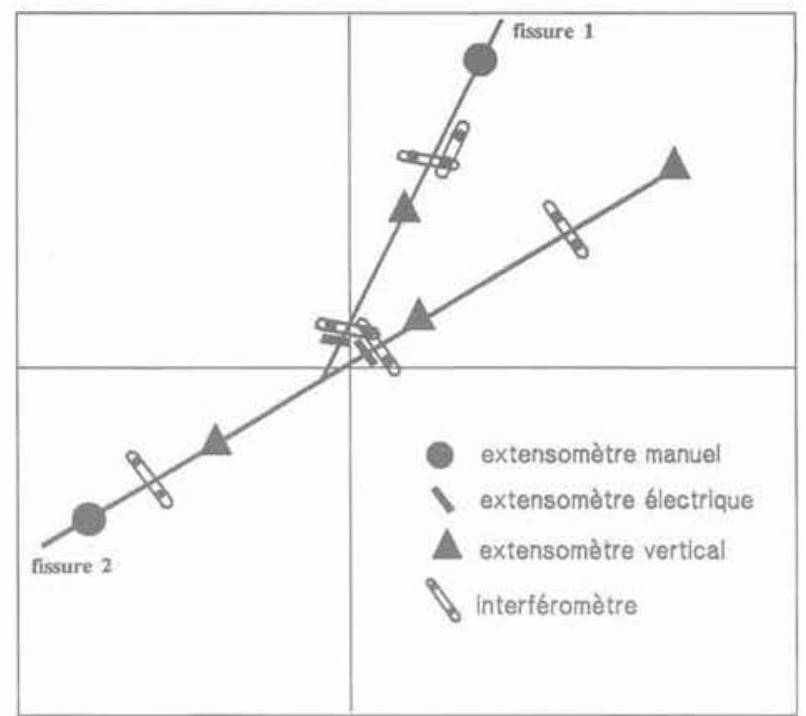

Fig. 7. - Instruments de mesure au niveau des 2 fissures principales du radier. Fig. 7. - Measuring devices on the 2 main fractures of the footwall.

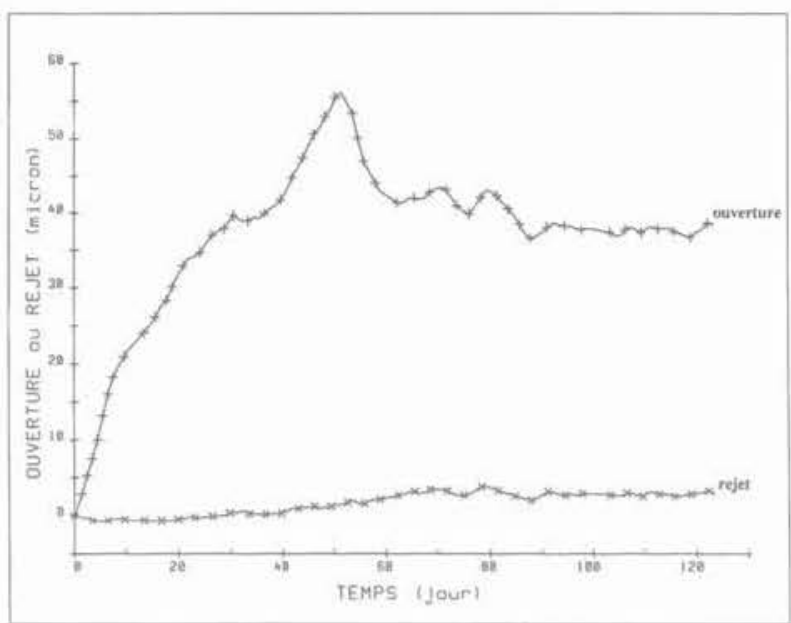

Fig. 8. - Résultats des mesures d'ouverture et de rejet de la fissure 2.

Fig. 8. - Measurement of opening and throw of fracture 2.

En ramenant à deux dimensions le problème de la simulation de l'expérience THM, un modèle en révolution avec une source de chaleur en forme de disque a été construit. La comparaison des mesures in situ aux résultats des divers modèles établis dans une étude de prédimensionnement de la source (VOUIL. LE, 1986) a montré que c'est le modèle bidimensionnel qui est le plus convenable pour simuler la géométrie réelle de l'essai. Les dimensions et les limites de ce modèle axisymétrique ainsi que le maillage correspondant sont donnés par la figure 9. Ce maillage, suffisamment fin à proximité de la source où le flux thermique est important, comporte 1098 nœuds et 507 éléments triangulaires quadratiques à 6 nœuds. Il a été construit de sorte que chaque point de mesure coïncide avec un nœud du maillage, ce qui facilite la comparaison entre les valeurs numériques et expérimentales. 


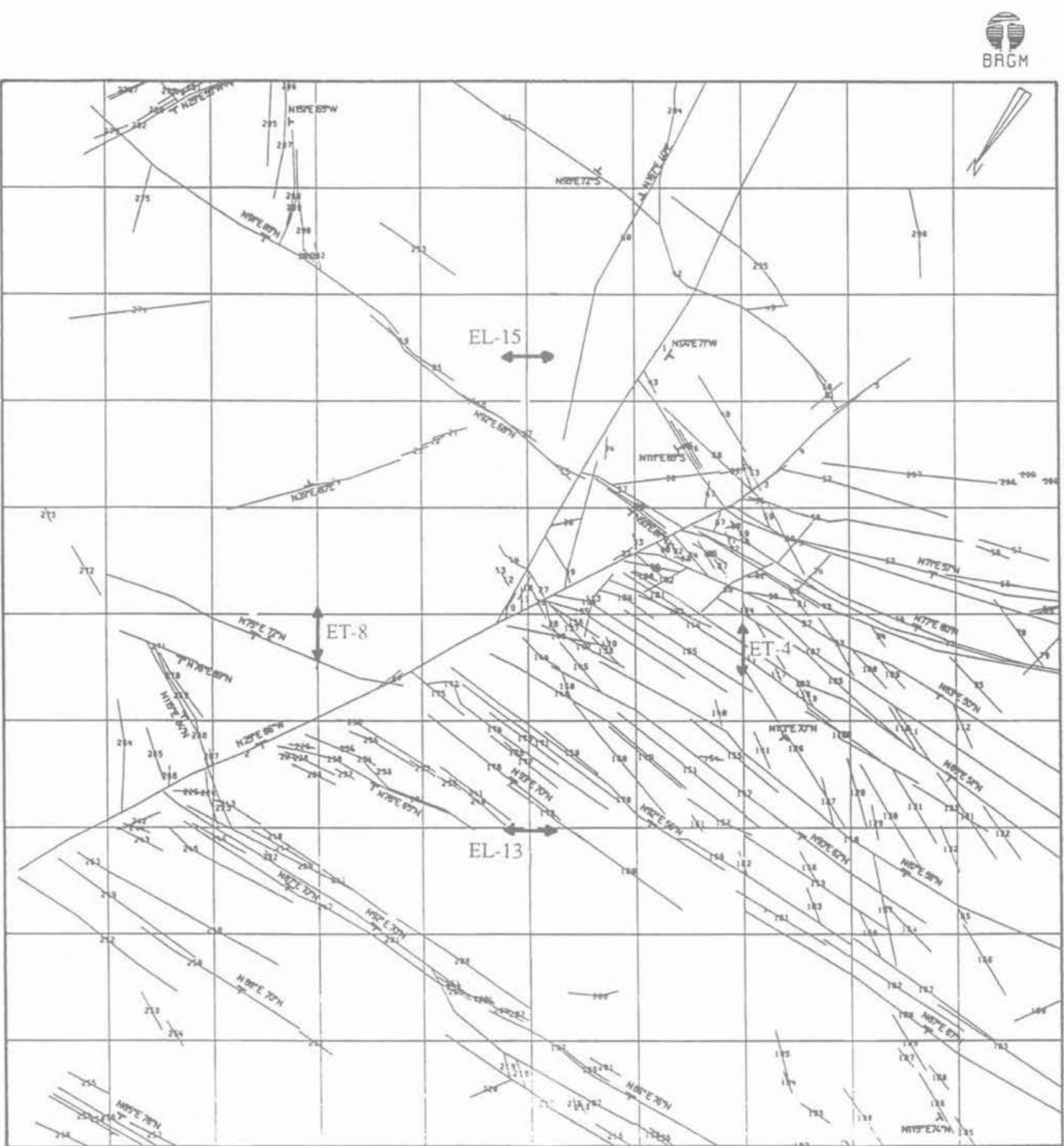

Fig. 6. - Position des extensométres de la figure 5 par rapport aux fissures du radier. Fig. 6. - Location of the extensometers of figure 5 in relation to the fractures of the footwall.

\section{Simulation thermique}

L'équation de la chaleur a été résolue avec les conditions aux limites suivantes:

- la paroi de la cavité $\left(\Gamma_{1}\right)$ simulant la source, est soumise à un flux constant correspondant à une puissance de chauffe de $1 \mathrm{~kW}$ s'exercant sur une surface équivalente de $5,61 \mathrm{~m}^{2}$ (surface d'un disque de $1,7 \mathrm{~m}$ de diamètre et de $0.2 \mathrm{~m}$ de hauteur), environ égale à celle de la source réelle:
- les parois de la chambre d'essai $\left(\Gamma_{3}\right)$ sont soumises à la température ambiante, supposée décroissante de $13{ }^{\circ} \mathrm{C}$ à $10^{\circ} \mathrm{C}$ au bout de 120 jours ; cette condition est inspirée des résultats de mesures des capteurs de température à la surface du radier;

- sur les limites $\Gamma_{2}$ (axe de symétrie), $\Gamma_{4}, \Gamma_{5}$ et $\Gamma_{6}$, nous avons imposé un flux nul.

L'état initial est défini par un champ de température uniforme et isotrope dans tout le massif égal à $13^{\circ} \mathrm{C}$, qui représente une valeur moyenne des températu- 


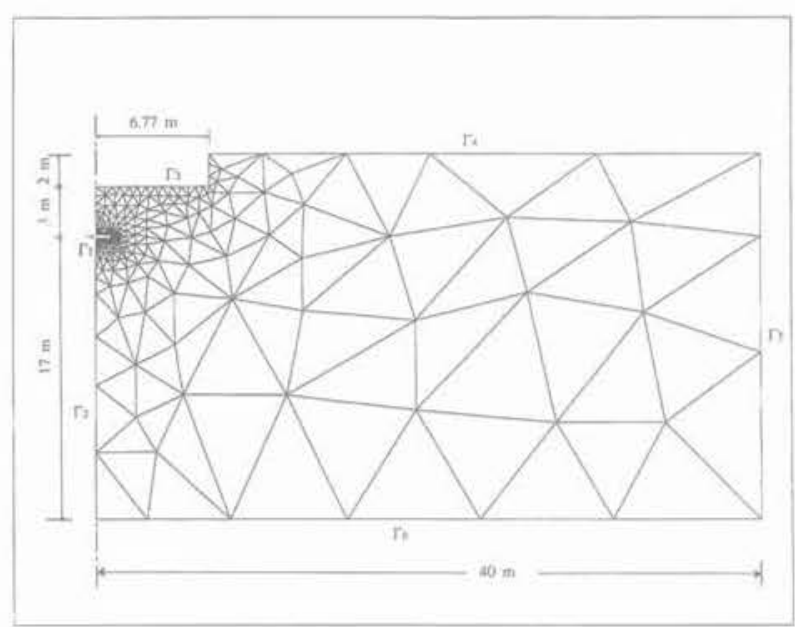

Fig. 9. - Maillage et limites du modèle.

Fig. 9. - Mesh and boundaries of the model.

res initiales mesurées in situ. Pour se rapprocher davantage des conditions réelles de l'expérience, nous avons considéré une durée de chauffage de 51 jours 21 heures 30 minutes, exactement égale à celle de l'expérience. Nous avons aussi simulé les deux premières pannes de chauffage, qui ont montré une influence significative sur les mesures, en ramenant et en maintenant à zéro le flux durant l'interruption.

La simulation a été effectuée en utilisant les caractéristiques thermiques du granite de Fanay-Augères, déterminées au laboratoire, en tenant compte de leur variation avec la température (BERGUES et $\mathrm{HABIB}$, 1988), à savoir :

$$
\begin{aligned}
\lambda(\mathrm{T}) & =4,725 / \mathrm{T}+1,9 \\
\mathrm{C}_{\mathrm{p}}(\mathrm{T}) & =1,45 \mathrm{~T}+770
\end{aligned}
$$

où :

$\lambda$ est la conductivité thermique en $\mathrm{W} / \mathrm{m} /{ }^{\circ} \mathrm{C}$,

$\mathrm{C}_{\mathrm{p}}$ est la chaleur spécifique en $\mathrm{J} / \mathrm{kg} /{ }^{\circ} \mathrm{C}$,

$\mathrm{T}$ est la température en ${ }^{\circ} \mathrm{C}$.

\section{Simulations thermomécaniques}

Deux calculs thermomécaniques ont été effectués en vue d'interpréter l'ensemble des mesures de déformations obtenues in situ; ces deux calculs utilisent l'historique des températures donné par la précédente simulation thermique.

Premier calcul : c'est un calcul thermoélastique de référence basé sur l'hypothèse d'un milieu continu. Dans ces conditions, les variations de contraintes sont reliées aux variations de température par un opérateur linéaire et les déformations sont proportionnelles aux contraintes thermiques. Cette hypothèse fait donc abstraction du rôle des fissures qui pourrait éventuellement modifier le comportement du massif rocheux ; c'est précisément la mise en évidence de ce rôle qui constitue l'objectif principal de ce calcul de référence.

Deuxième calcul : la référence étant connue, un calcul thermoélastoplastique est réalisé dans le but d'introduire le rôle de la fissuration moyennant l'utilisation d'un milieu continu isotrope équivalent caracté- ristisé par une résistance en traction nulle (REJEB et VOUILLE, 1989).

Les deux calculs élastique et plastique diffèrent uniquement par la loi de comportement utilisée. Celle du matériau à traction nulle représente le cas extrême où les fissures s'ouvrent sans se fermer pendant la décharge.

Les conditions aux limites auxquelles a été soumis le modèle portent sur le champ de déplacement:

- déplacement horizontal nul sur $\Gamma_{2}$ et $\Gamma_{5}$ :

- déplacement vertical nul sur $\Gamma_{4}$ et $\Gamma_{6}$.

Les conditions initiales sont définies par les pressions verticale $\mathrm{P}$ et horizontale $\mathrm{Q}$ dues au poids des terres. Le coefficient de proportionnalité entre les contraintes in situ a été pris égal à 1 conformément aux résultats des essais réalisés sur le site considéré (FEUGA et OUVRY, 1988) ; soit alors $\mathrm{P}=\mathrm{Q}=\gamma \mathrm{h}=2,6 \mathrm{MPa}$, où :

$\gamma$ est le poids volumique du granite de Fanay égal à $26 \mathrm{kN} / \mathrm{m}^{3}$,

$\mathrm{h}$ est la hauteur du terrain de recouvrement au-dessus de la chambre d'essai égale à $100 \mathrm{~m}$.

Les paramètres thermomécaniques utilisés dans ces simulations sont ceux du granite de Fanay-Augères testé au laboratoire (VOUILLE et HUMBERT, 1988) :

Module d'Young : $\mathrm{E}=55650 \mathrm{MPa}$,

Coefficient de Poisson : $\nu=0,22$,

Coefficient de dilatation thermique : $\alpha=5.10^{-6}{ }^{\circ} \mathrm{C}^{-1}$,

\section{DISCUSSION}

Pour la validation de notre modélisation thermomécanique, les résultats numériques ont été comparés aux résultats des mesures de température et de déformation.

\subsection{Répartition des températures}

La comparaison entre la simulation thermique et l'expérience a été effectuée dans l'espace et dans le temps respectivement à l'aide des isothermes et des courbes représentant l'historique des températures (REJEB et al., 1988), dont trois exemples sont illustrés sur les figures 10, 11 et 12 .

L'analyse de l'ensemble des résultats nous a permis de dégager les points suivants :

- les résultats du calcul sont en bon accord avec les mesures ;

- dans lensemble, le modèle sur-estime légèrement les températures et en particulier aux ncuds les plus proches de la source (fig. 10);

- l'accord entre le calcul et les mesures s'améliore au fur et à mesure qu'on s'éloigne de la source puisque linfluence de la différence de forme entre la 


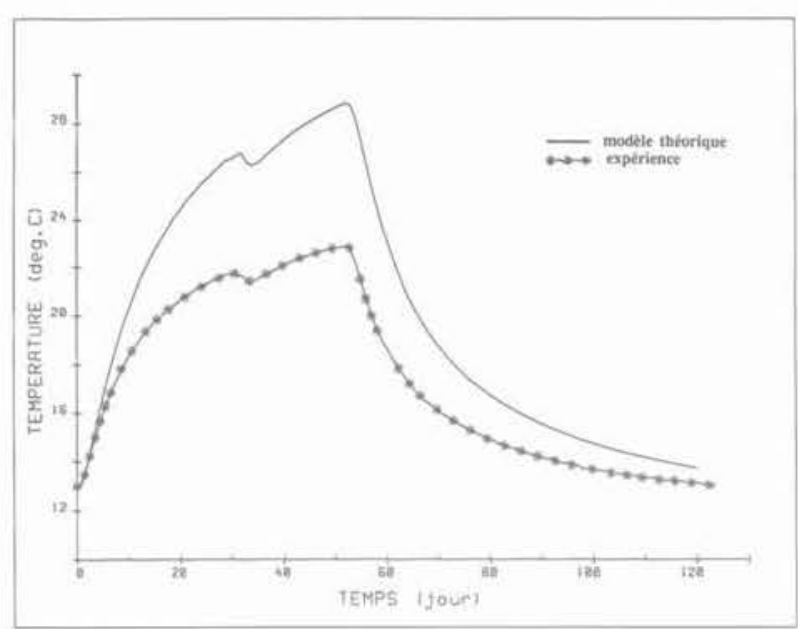

Fig. 10. - Historique des températures calculées et mesurées d̀ $1 \mathrm{~m}$ de la source de chaleur.

Fig. 10, - Evolution vs time of the temperatures computed and measured at $1 \mathrm{~m}$ from the heat source.

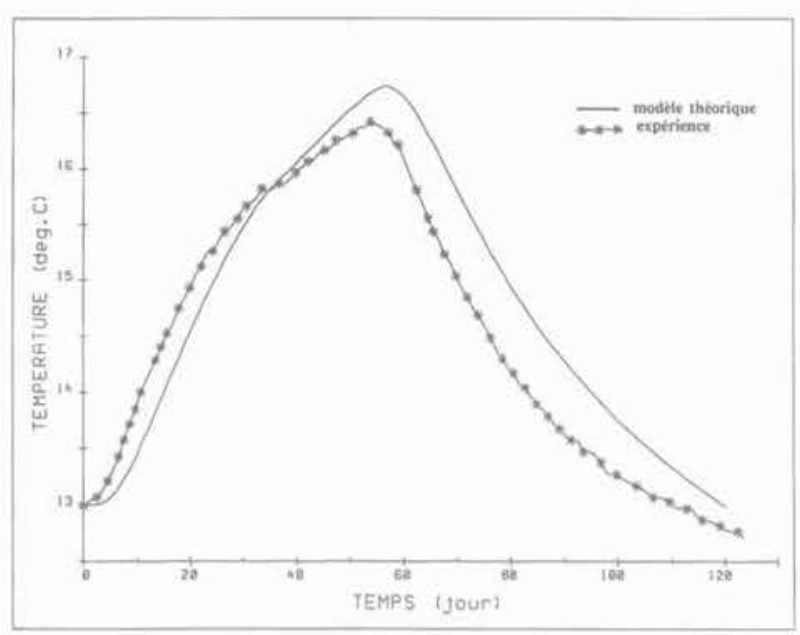

Fig. 11. - Historique des températures calculées et mesurées à $3 \mathrm{~m}$ de la source de chaleur.

Fig. 11. - Evolution vs time of the temperatures computed and measured at $3 \mathrm{~m}$ from the heat source.

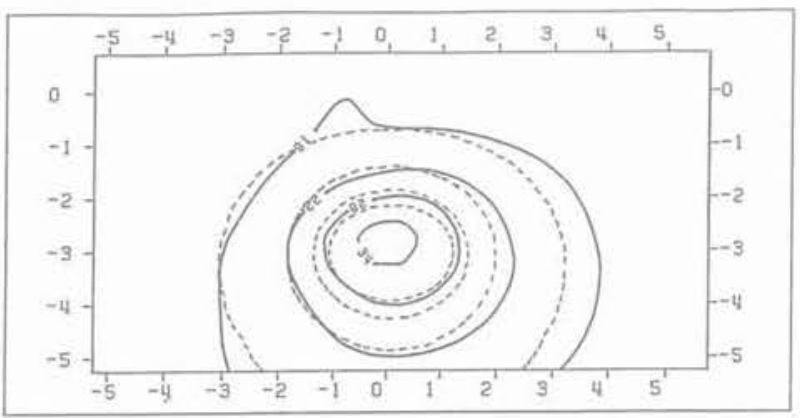

Fig. 12. - Isothermes théoriques (..) et expérimentales (-) à la date 51 jours.

Fig. 12. - Theoretical ( ..-) and experimental $(-)$ isothermals on day 51.

source réelle (5 éléments cylindriques) et simulée (disque) devient négligeable (fig. 11) ;
- l'écart relativement important entre les lignes isothermes $\left(34^{\circ} \mathrm{C}\right)$ théorique et expérimentale de la figure 12 est dû, d'une part à la raison précédemment mentionnée concernant la forme de la source et, d'autre part au fait que nous n'avons pas tenu compte des mesures des températures de la source lors de la construction des isothermes expérimentales; en effet, la présence de ces températures élevées au centre devrait probablement dilater davantage les isothermes expérimentales au voisinage de ce point.

\subsection{Déformations du massif}

Les résultats des calculs élastique et élastoplastique sont confrontés successivement aux mesures en profondeur et en surface.

\section{Déplacement vertical en profondeur}

Les figures 13,14 et 15 donnent chacune un exemple de résultats obtenus dans l'un des trois forages $\mathrm{F}_{1}, \mathrm{~F}_{2}, \mathrm{~F}_{3}$; on y a représenté en fonction du temps le déplacement relatif par rapport à la surface de points d'ancrage respectivement situés à $4 \mathrm{~m}, 4 \mathrm{~m}$ et $3 \mathrm{~m}$ de profondeur. Pour une meilleure interprétation de ces courbes, nous considérons séparément les deux phases de chauffage et de refroidissement.

Période de chauffage

Les prévisions du calcul élastoplastique sont en meilleur accord avec les mesures des extensomètres des forages $F_{1}$ et $F_{2}$ que celles du calcul élastique (fig. 13 et 14). Dans le cas du forage $\mathrm{F}_{3}$, la situation s'inverse et un écart assez important est observé entre les résultats du milieu équivalent et les mesures in situ (fig. 15) ; ce qui laisse supposer soit l'absence de fissures horizontales dans la zone de ce forage, soit la présence

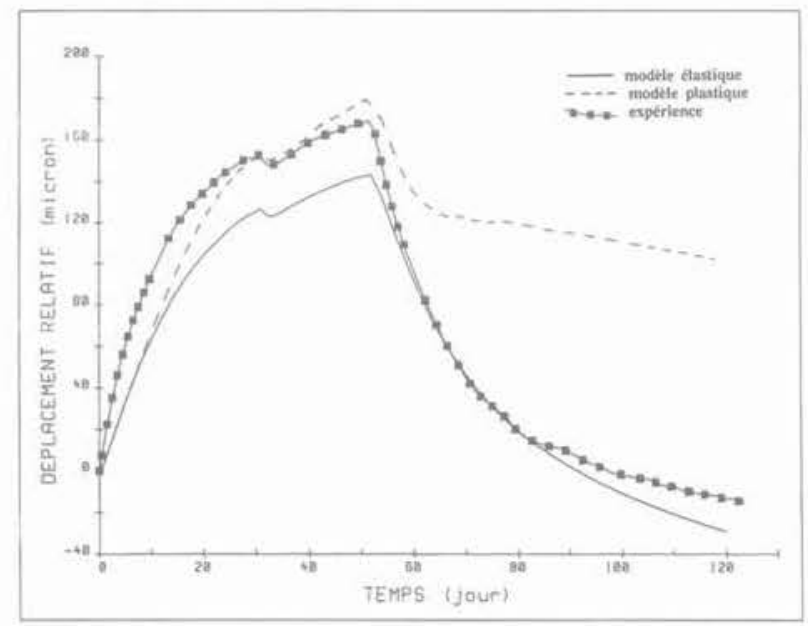

Fig. 13. - Comparaison des calculs aux mesures de l'extensomètre EF-13, dans le forage $F_{1}$ à $1 \mathrm{~m}$ de la source de chaleur.

Fig. 13. - Comparison between calculations and extensometer EF-13 measurements, in the borehole $F$, at $1 \mathrm{~m}$ from the heat source. 


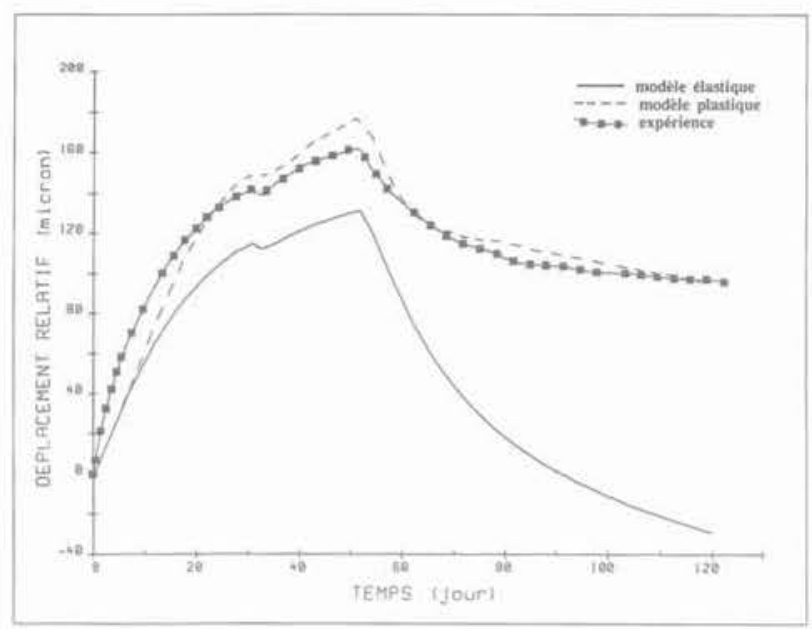

Fig. 14. - Comparaison des calculs aux mesures de l'extensomètre EF-24, dans le forage $F_{2}$ a $1,41 \mathrm{~m}$ de la source de chaleur.

Fig. 14. - Comparison between calculations and extensometer EF-24 measurements, in the borehole $F_{2}$ at $1.41 \mathrm{~m}$ from the heat source.

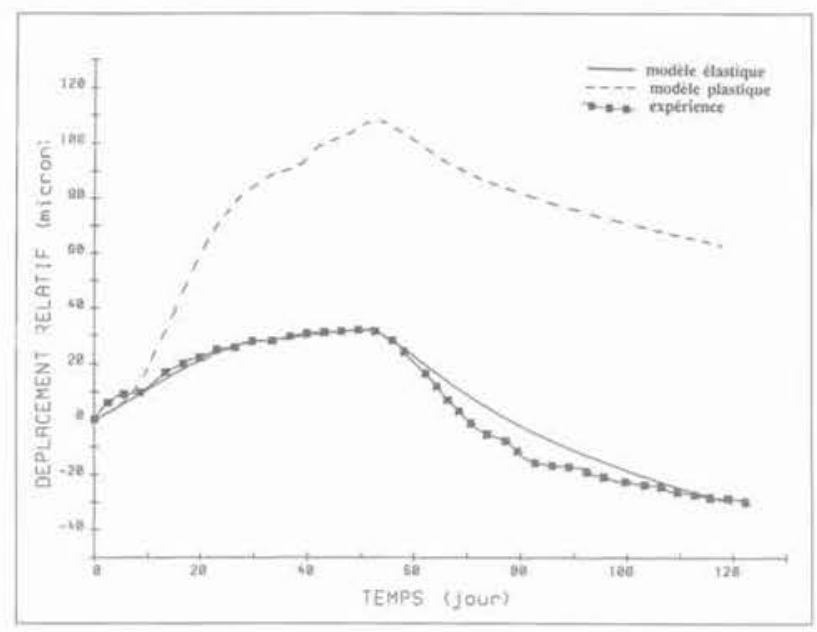

Fig. 15. - Comparaison des calculs aux mesures de l'extensomètre EF-33, dans le forage $\mathrm{F}_{3}$ à $3 \mathrm{~m}$ la source de chaleur.

Fig. 15. - Comparison between calculations and extensometer EF-33 measurements, in the borehole $F_{3}$ at $3 \mathrm{~m}$ from the heat source.

de fissures qui ne sont pas ouvertes pendant le chauffage.

Nous interprétons le grand écart entre le premier et le deuxième calcul au niveau du forage $F_{3}$ par le fait que la position de ce forage est au centre de la zone plastique qui s'est développée pendant le chauffage (fig. 16). Ainsi lors du calcul élastoplastique, les nœuds correspondants aux points d'ancrage du forage $\mathrm{F}_{3}$ subissent en plus des déformations élastiques et thermiques, d'importantes déformations irréversibles. Par ailleurs, le faible écart entre les deux calculs au niveau des forages $F_{1}$ et $F_{2}$ est da au fait qu'ils sont situés pratiquement dans une zone élastique (fig. 16) où les déformations plastiques, qui auraient pu différencier les deux résultats, sont presque nulles. Cepen-

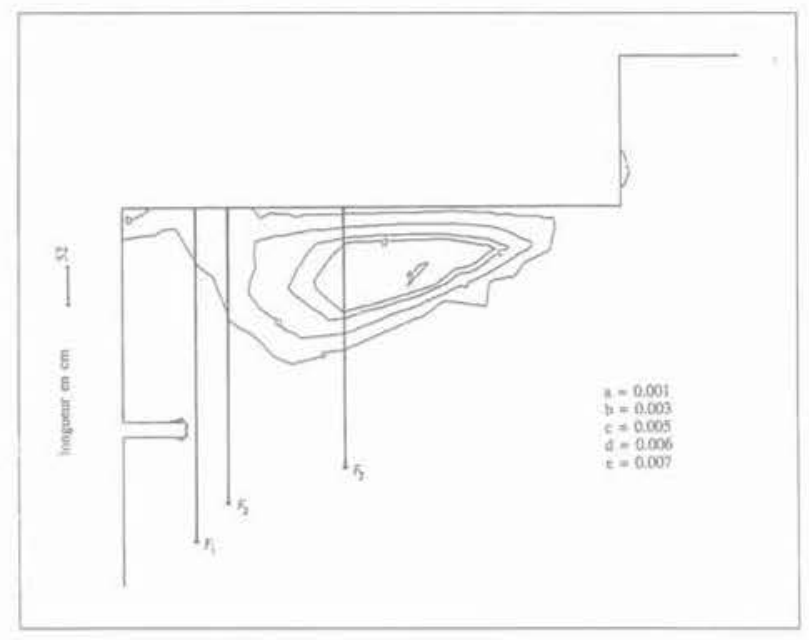

Fig. 16. - Position des forages par rapport aux lignes isovaleurs de la norme de la déformation plastique, à 51 jours.

Fig. 16. - Location of the boreholes

in relation to the isolines of the plastic deformation norm, on day 51.

dant, ce faible écart montre que les reports de charges à distance provoqués par la fissuration sont peu importants.

\section{Période de refroidissement}

Les prévisions du calcul élastique sont en bon accord qualitatif et quantitatif avec les mesures de tous les extensomètres de forage, exception faite de l'extensomètre EF-24 du forage $F_{2}$. En revanche, l'excellent accord obtenu entre les mesures provenant de cet extensomètre EF-24 et les résultats du calcul élastoplastique (fig. 14) confirme la possibilité d'existence d'une fissure horizontale entre les profondeurs - $3,5 \mathrm{~m}$ et $-4 \mathrm{~m}$, qui s'est ouverte pendant le chauffage sans se refermer au cours du refroidissement.

Les déformations du matériau sans traction au niveau des trois forages sont nettement plus importantes que celles du matériau élastique et que celles mesurées in situ. Cela provient de l'irréversibilité de la déformation plastique pendant le déchargement. En revanche, les déformations thermiques et élastiques sont réversibles et c'est cette réversibilité qui explique la légère contraction que lon peut constater sur les courbes obtenues par le calcul élastoplastique pendant les 15 premiers jours de refroidissement.

L'analyse des résultats du modèle plastique montre que le comportement irréversible observé pendant le refroidissement sur les historiques du déplacement relatif provient de l'irréversibilité du déplacement vertical des nœeuds à la surface du radier (zone plastique) et non pas des nœuds en profondeur (zone élastique).

Notons que c'est à cause de la variation de la température extérieure imposée dans le calcul thermique que le massif continue à se déformer, dans le cas du calcul élastique, pendant le refroidissement au-delà de son état initial de déformation nulle. 
Durant le chauffage et le refroidissement, l'écart entre l'expérience et les simulations thermomécaniques est en partie expliqué par la répercussion sur le phénomène mécanique de l'écart observé auparavant entre les températures mesurées et calculées.

\section{Déformations en surface}

Les déformations tangentielles et radiales, calculées à partir du champ de déplacements, sont comparées aux mesures des extensomètres électriques longitudinaux et transversaux. La figure 17 illustre un exemple de comparaison entre la déformation tangentielle calculée et mesurée à $2 \mathrm{~m}$ du centre du radier. On constate que les résultats théoriques sont globalement proches des mesures de l'extensomètre EL-15 se trouvant dans une zone du radier, relativement non fissurée (fig. 6). On remarque aussi que les deux modèles donnent des déformations tangentielles $\left(\epsilon_{\theta}\right)$ pratiquement égales ; en effet les résultats du modèle plastique n'ont pas montré d'importantes ouvertures de fissures perpendiculairement au radier qui auraient pu différencier le déplacement horizontal (U) et par suite $\epsilon_{\theta}$ dans les deux cas de calcul, puisque celle-ci est calculée à partir de la formule $\epsilon_{\theta}=\mathrm{U} / \mathrm{r}$, où $\mathrm{r}$ est l'abscisse du point considéré.

Par ailleurs, l'écart entre les deux résultats théoriques est plus important dans le cas des déformations radiales à $4 \mathrm{~m}$ du centre du radier comme le montrent les courbes de la figure 18. Dans ce cas, on note que le calcul élastoplastique rend parfaitement compte de l'ensemble des mesures pendant le chauffage et particulièrement des mesures en phase de refroidissement de l'extensomètre ET-12 placé à cheval sur une fissure.

D'une manière générale, la confrontation des déformations théoriques en surface aux mesures in situ a montré un accord qualitatif acceptable mais de nettes différences sur les ordres de grandeur, le calcul sous-estime nettement l'ampleur des phénomènes observés. La divergence entre le modèle élastique et

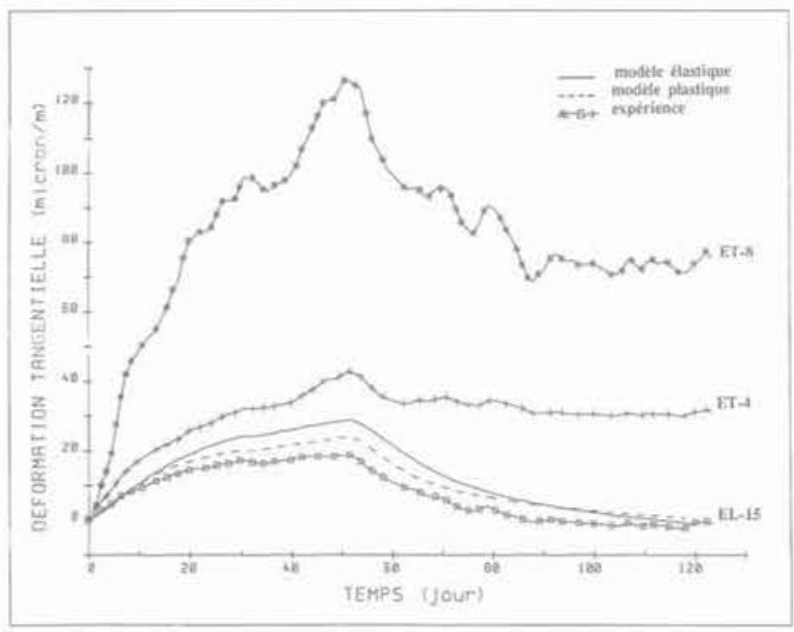

Fig. 17. - Comparaison entre les déformations tangentielles calculées et mesurées à $2 \mathrm{~m}$ du centre du radier.

Fig. 17. - Comparison between the tangentiel strains computed and measured at $2 \mathrm{in}$ from the footwall center.

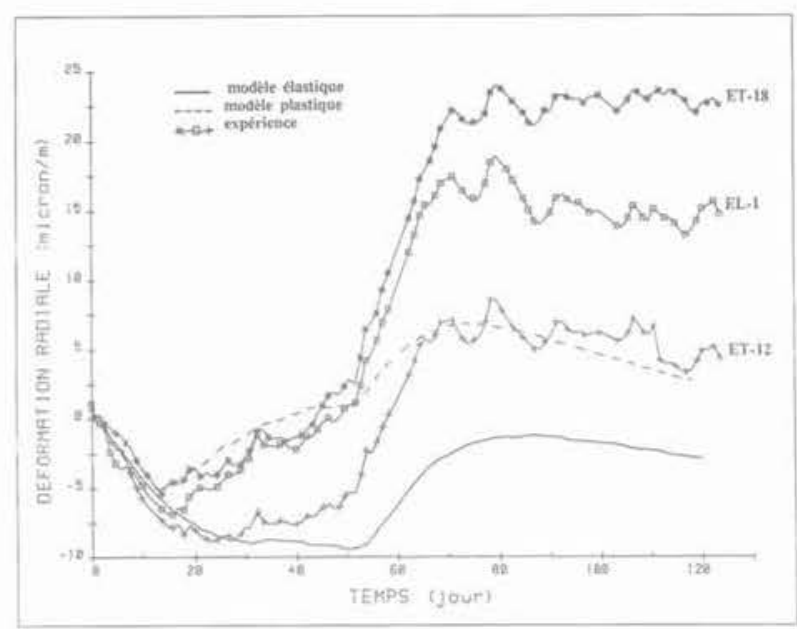

Fig. 18. - Comparaison entre les déformations radiales calculées et mesurées à $4 \mathrm{~m}$ du centre du radier.

Fig. 18, - Comparison between the radial strains computed and measured at $4 \mathrm{~m}$ from the footwall center

l'expérience s'accentue encore davantage dès que la tendance de l'évolution des déformations s'inverse, c'est-à-dire aussitôt après l'arrêt du chauffage. Cette divergence doit être attribuée à une ouverture irréversible des fissures qui interceptent le radier de la chambre d'essai, de ce fait celui-ci apparaît plus déformable que ne le serait un milieu continu et présente un tout autre comportement au cours de la phase de refroidissement.

Le calcul de référence a donc permis de confirmer l'effet significatif de la fissuration évoqué dans le paragraphe 2. Cet effet s'est traduit dans un premier temps par une augmentation des déformations des zones fissurées, de moindre rigidité, et dans un second temps par une irréversibilité du comportement assez marquée peut être due au jeu des fissures.

\section{CONCLUSION}

Le dépouillement et le traitement des mesures thermomécaniques du laboratoire souterrain de FanayAugères nous ont permis de constituer une importante base expérimentale; cette base doit être le point d'ancrage de toute modélisation.

L'interprétation thermique a montré que le transfert de chaleur dans le granite fracturé du laboratoire THM obéit à la loj de Fourier. En effet, l'uniformité et la cohérence de la répartition du champ de température montrent que la présence des fissures n'a pas eu un effet important sur les mesures. De plus, en utilisant les conditions réelles de l'expérience, la modélisation du comportement thermique du massif avec l'hypothèse d'un milieu continu isotrope a donné des résultats satisfaisants permettant de rendre compte des mesures in situ. Le faible écart observé entre la théorie et l'expérience est da principalement au caractère bidimensionnel du modèle réalisé. Nous pensons qu'une représentation en trois dimensions de la géométrie 
réelle de l'essai et de la source de chaleur pourrait améliorer les résultats numériques.

Par ailleurs, l'interprétation des mesures de déformation en surface et en profondeur a révélé un effet significatif de la fissuration qui nous paraît être lié à l'anisotropie directionnelle du système de fractures qui affecte le massif et à l'existence de fractures majeures individualisées, compartimentant le radier de la chambre d'essai.

L'utilisation d'un matériau à traction nulle en milieu continu équivalent s'est avérée parfois insuffisante à cause de l'irréversibilité totale des déformations plastiques. Cela démontre la nécessité d'introduire au niveau de cette loi « sans traction * une certaine réversibilité, observée sur les mesures d'ouverture de fissures, afin de pouvoir affiner la comparaison et $\approx \mathrm{col}$. ler * à la réalité des observations expérimentales.

Compte tenu du fait que c'est parfois la loi de comportement élastique et parfois la loi * sans traction " qui simule le mieux le comportement du granite fracturé, il nous semble que la solution résiderait dans une modélisation où le domaine étudié serait divisé en plusieurs zones caractérisée chacune par un comportement mécanique spécifique (matériau intact, matériau à fracturation diffuse isotrope, matériau à fracturation diffuse anisotrope, etc.) et séparées par des joints plans dont on modéliserait le comportement mécanique (cohésion, angle de frottement, dilatance, etc.). Une telle modélisation nécessiterait évidemment une représentation tridimensionnelle du domaine étudié.

\section{REMERCIEMENTS}

Les auteurs remercient l'Institut de Protection et de Sûreté Nucléaire du Commissariat à l'Energie Atomique pour son soutien financier.

\section{BIBLIOGRAPHIE}

BERGUES J., HABIB P. (1988), Etude de la fissuration du granite d'Auriat; Mesure de la conductivité thermique du granite de Fanay. Rapport $n^{\circ} 5$, Ecole Polytechnique, LMS, France, Oct. 1988.

CCE (1982), Sciences et techniques nucléaires ; La charge thermique admissible en formations géologiques: Conséquences sur les méthodes d'évacuation des déchets radioactifs. Rapport EUR 8179 EN/FR, Volume 1.

CCE et OCDE-AEN (1984), Evacuation des déchets radioactifs en formations géologiques: panorama de létat actuel des connaissances. Rapport d'experts préparé par la CCE et l'OCDE-AEN.

CEA, IPSN-SEPD (1986), Etude des possibilités de stockage dans les formations géologiques. Rapport CEA/IPSN/SEPD n 86/01, Février 1986.
CÔME B., JOHNSTON P., MÜLLER A. (1985), La conception et l'instrumentation d'expériences in situ en laboratoires souterrains pour l'évacuation des déchets radioactifs. Comptes rendus d'une séance de travail CCE-AEN, Mai 1984.

CÔME B (1988), Aspects thermomécaniques liés à lévacuation géologique des déchets radioactifs. La Thermomécanique des Roches, Manuel et Méthodes $n^{\circ} 16$, pp. 158-191, Editions BRGM, Orléans, France.

FEUGA B., OUVRY J.F. (1988), Laboratoire souterrain dans le granite de Tenelles-mine de Fanay; Expérience thermo-hydro-mécanique; Détermination des contraintes in situ par sollicitation hydraulique dans les sondages S2, S3 et S4. Rapport du BRGM, 88 SGN 405 STO, Mai 1988.

GATHION E., PINCENT P. (1988), Etude des possibilités de stockage dans les formations géologiques, Rapport CEA/IPSN/SEPD n०88/24, Annexes 2 et 3, Décembre 1988.

OCDE-AEN (1983), L'évacuation des déchets radioactifs dans des formations granitiques: expérience in situ dans du granite. Comptes rendus d'une réunion de travail de l'AEN, Stockholm, Oct. 1982.

REJEB A., CHARLET B., VOUILLE G. (1988), Laboratoire Thermo-Hydro-Mécanique : Mine de Fanay-Augères ; Dépouillement et interprétation des mesures thermiques. Rapport R 88/21. ENSMP/CGES/MR, Fontainebleau, Sept. 1988.

REJEB A., CHARLET B., VOUILLE G. (1989), Laboratoire Thermo-Hydro-Mécanique: Mine de Fanay-Augères ; Traitement et interprétation des mesures thermomécaniques. Rapport R 89/1. ENSMP/CGES/MR, Fontainebleau, Janv. 1989.

REJEB A., VOUILLE G. (1989), Résultats préliminaires des simulations thermoélastoplastiques: Recherche d'un milieu continu équivalent. Rapport R $89 / 21$, ENSMP/CGES/MR, Fontainebleau, Sept. 1989.

TIJANI S.M. (1985), Les logiciels CHEF et VIPLEF ; Notices d'utilisation. ENSMP/CGES/MR, Fontainebleau.

VOUILLE G. (1986), Etude des possibilités de stockage dans les formations géologiques. Rapport CEA/IPSN/SEPD n 86/01. Annexes III et IV. « Etude de dimensionnement de la source chauffante », Février 1986.

VOUILLE G., HUMBERT B. (1988), Fanay-Augères, Caractéristiques thermomécaniques du granite. Rapport SE 88/3, ENSMP/CGES/MR, Fontainebleau, Avril 1988.

ZIMMERMAN R.M. (1986), NNWSI Project: G. Tunnel heated block experiment. Rapport Sandia $n^{\circ}$ SAND 84-2620, Mai 1986. 